\title{
Prostate artery embolisation: an initial experience from an Indian perspective
}

\author{
Soumil Singhal, Bibin Sebastian, Rohit Madhurkar, M C Uthappa \\ BGS Gleneagles Global Hospital, Bangalore, India
}

\section{Abstract}

Purpose: The study aims to report our very early experience with prostate artery embolisation in patients with benign prostatic hypertrophy (BPH) in an Indian setting.

Material and methods: This prospective study was approved by the Institutional Ethical Review Board, and informed consent was obtained from all the patients. Four patients with lower urinary tract symptoms due to benign prostatic hyperplasia underwent prostate embolisation. Embolisation was performed using polyvinyl alcohol (PVA) particles. International Prostate Symptom Score (IPSS), quality of life (QOL) score, postvoid residual volume, and prostate volume was recorded in each patient for 12 months. No major complications were seen in any patient.

Results: Four patients with a mean age of 74.2 years underwent prostate artery embolisation. It was successful in all the cases. Bilateral embolisation was performed in three patients and unilateral approach in one patient. Clinical improvement was characterised by a mean prostate volume reduction of $22 \%$ and mean IPSS reduction of $30 \%$ at 12-month follow-up.

Conclusions: The initial experience with prostate embolisation has been very rewarding and is a great alternative treatment for BPH. It is a very safe and effective procedure and can be an ideal procedure from an Indian perspective.

Key words: embolization, prostate, angiography.

\section{Introduction}

Benign prostatic hyperplasia $(\mathrm{BPH})$ is a common problem in men, especially among the geriatric group. It is very troublesome because of typical lower urinary tract symptoms (LUTS) [1]. LUTS are characterised by frequency, intermittency, and urgency along with incomplete bladder emptying sensation, straining, poor stream, frequent trips to the toilet in the night, and sexual dysfunction. All of these have a significant negative impact on quality of life [2]. It is concluded that about $50 \%$ of men above the age of 50 years suffer from LUTS. In the age group of 70-89 years, this is as high as $90 \%[1,3]$. In a country like India, where the life expectancy is improving, the prevalence of $\mathrm{BPH}$ is showing an upward trend.
Medical management is the first choice of treatment for moderate LUTS due to BPH [4]. However, on long-term follow-up patients show resistance to medical management, requiring transurethral resection of the prostate (TURP) or open surgery. TURP is a gold standard procedure, which is highly effective in symptom reduction in BPH [5]. Even though there is excellent symptomatic improvement, TURP is associated with complications like haemorrhage, urinary tract infections, transurethral resection (TUR) syndrome, urethral stenosis, urinary incontinence or retention, sexual dysfunction, retrograde ejaculation, and post-operative pain [6]. Development of bipolar TURP [7] and other minimally invasive techniques like laser prostatectomy and microwave ablation have reduced these complications, especially blood loss and TUR syndrome. However, these

Correspondence address:

Dr. Bibin Sebastian, BGS Gleneagles Global Hospital, Department of Interventional Radiology and Intervent, 560060, Bangalore, India,

e-mail: drbibinsebastian@gmail.com

Authors' contribution:

A Study design · B Data collection · C Statistical analysis · D Data interpretation - E Manuscript preparation · F Literature search · G Funds collection 


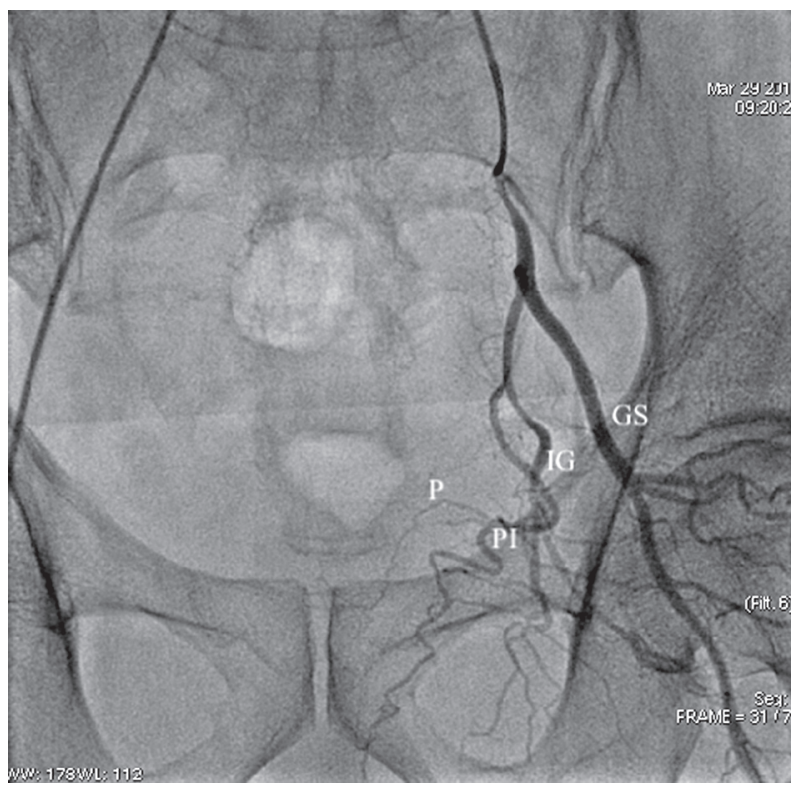

Figure 1. Pelvic angiography for identification of prostate arteries. Note the following branches: superior gluteal artery (GS), inferior gluteal artery (IG), internal pudendal artery $(\mathrm{PI})$, and prostate artery $(\mathrm{P})$

techniques are usually costly, not readily available, and not very practical. There are no long-term data available on the outcome [8].

Prostate artery embolisation (PAE) is a safe and effective treatment for $\mathrm{BPH}$, which is based on the similar principle of uterine artery embolisation for uterine fibroids [9]. Prostate artery embolisation is gaining popularity in the western world. There is greater awareness and acceptance among the clinicians and patients in recent times. It was pioneered by DeMeritt et al., who first performed embolisation of the prostate for management of postbiopsy haematuria in a case of BPH in the year 2000 [10] and by Carnevale et al. who performed the first PAE for $\mathrm{BPH}$ and demonstrated symptomatic improvement and volume reduction of the prostate in two patients in 2010 [11]. Recently the National Institute for Health and Care Excellence (NICE) recommended PAE for selected men with BPH based on the UK ROPE study (UK Register for Prostate Embolisation). The UK ROPE study concluded that PAE provides statistically significant and clinically evident improvement in symptoms and quality of life in patients with $\mathrm{BPH}[12]$.

This study is a demonstration of our initial experience in PAE for BPH with a technical account and outcome follow-up.

\section{Material and methods}

This prospective study was approved by the Institutional Ethical Review Board. The study was carried out between March 2016 to November 2017. Informed consent was taken from all the patients. Four patients with a mean age of 74.2 years (range: 58-91) underwent prostate ar- tery embolisation. All patients who underwent the procedure were refractory to medical management and were advised for surgery. Patients' clinical symptoms of the lower urinary tract were evaluated using the International Prostatic Symptoms Score (IPSS) and the Quality of Life (QOL) Questionnaire. Prostate diameters, volume, and post-void residual volume were assessed both pre- and post-embolisation by transabdominal ultrasonography. Serum PSA levels were measured in all cases. The embolisation technique was explained in detail to all patients. Pre-embolisation computed tomography (CT) angiogram was performed on a 64-row detector scanner (GE Medical Systems, Milwaukee, Wis) for procedure planning. Our angiography protocol included: kV 100-120 kV and 150-250 mA, $512 \times 512$-pixel matrix, collimation of $16 \times 0.6 \mathrm{~mm}$, and a pitch of 1.2 . Iodinated contrast material $(150 \mathrm{ml}$ at a concentration of $350 \mathrm{mg} / \mathrm{ml}$ iodine $)$ was injected at a rate of $4 \mathrm{ml} / \mathrm{s}$ by using bolus triggering in the abdominal aorta (above the renal arteries). Post-processing was performed using maximum intensity projections and volume rendering with three-dimensional reconstructions.

Patients were admitted one day before the procedure. Prostate embolisation was performed in the angiography suite (Innova 9100, GE Medical Systems, Milwaukee, Wis). The bladder was not catheterised. A single dose of Ceftriaxone was given intravenously prior to the procedure. Prostate artery embolisation was performed under local anaesthesia. Right femoral artery approach with local anaesthesia under ultrasound guidance and catheterised using a 5F RIM catheter (Cook, Bloomington, Indiana, USA). An angiographic run of the aorta followed by selective angiography of the internal lilac artery was performed to assess the prostate artery anatomy (Figure 1). Once the arterial anatomy was deciphered super-selective catheterisation was performed using a microcatheter (Progreat; Terumo, Tokyo, Japan). The position was confirmed following a check angiographic run (Figure 2). Embolisation was performed using non-spherical 180$300 \mu \mathrm{m}$ (Cook) polyvinyl alcohol particles. Slowing of the flow or near stasis in the prostate artery was chosen as an angiographic endpoint for the procedure. Bilateral prostate artery treatment was attempted in all cases (Figure 3). Post-procedure complications, if there were any, were documented according to Society of Interventional Radiologists classification [13]. Patients were discharged on the same day with antibiotics and non-steroidal antiinflammatory drugs for three days.

Patients were followed up at one month, six months, and 12 months by trans-abdominal ultrasound. Post-procedure IPSS and QOL were also recorded. No definite statistical analysis was done because this is a preliminary short-term follow-up study with a limited number of patients. Categorical variables are expressed as percentage, and continuous variables are expressed as mean \pm standard deviation. 

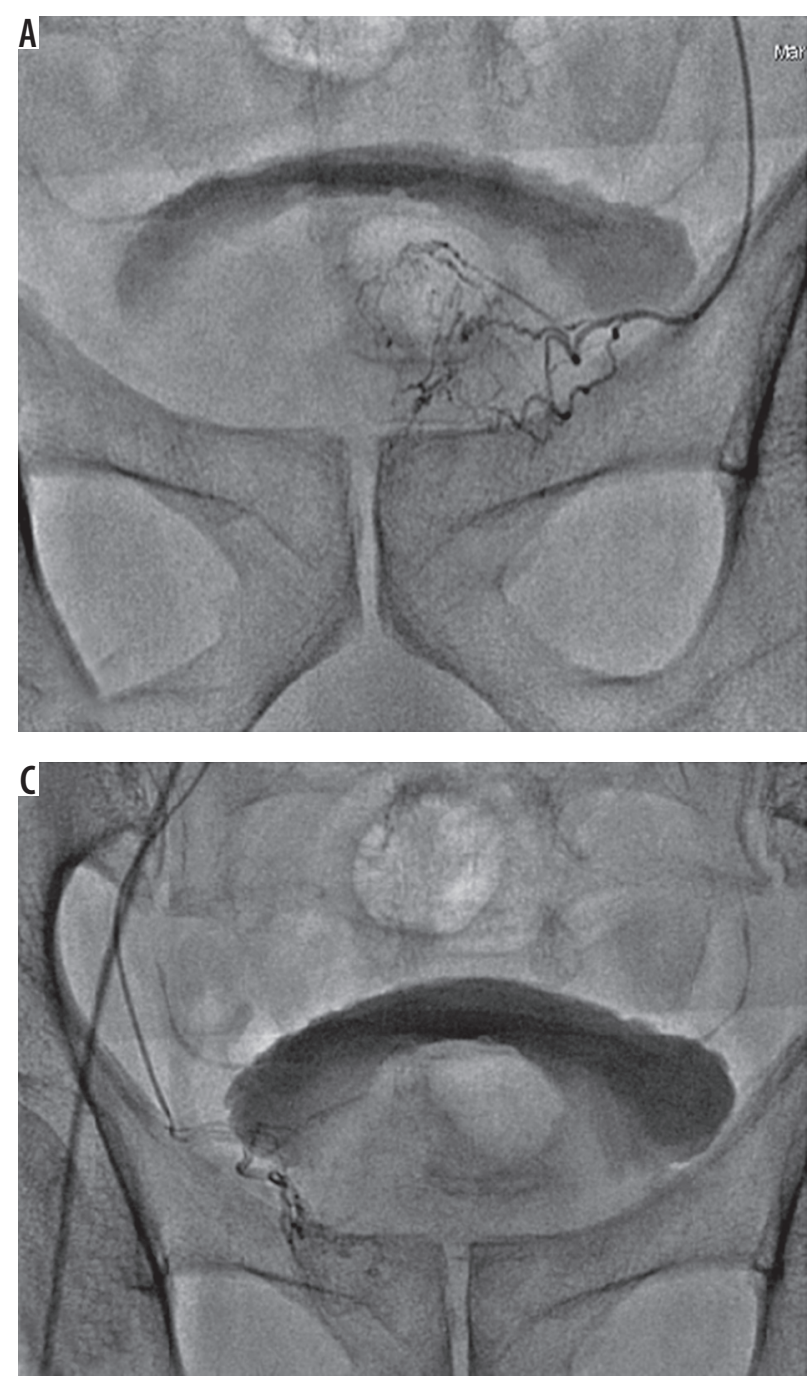

\section{Results}

Prostate artery embolisation was performed in four patients with a mean age of 74 years (range: 58-91). PAE was technically successful in all our cases (100\%). Bilateral embolisation was performed in three patients. A unilateral approach was adopted in one patient due to difficulty in the demonstration of the prostate artery.

Planning CT angiography was performed in all our patients to assess the anatomy. The right prostate artery had a Type A anatomy in four patients, and was seen arising from the internal pudendal artery in three and anterior trunk in one patient. The left prostate artery had Type A in three patients and Type B anatomy in one patient and was seen arising from the internal pudendal artery in all four patients. The mean prostate artery diameter noted was $1.8 \mathrm{~mm}$ on the right side and $2 \mathrm{~mm}$ on the left side. No procedure-related complications were noted. On comparing CT angiography versus convention angiography in detection and delineating the prostate artery anatomy $80 \%$ (three out of four cases) of prostate artery anatomy was well seen on CT angiography.

The procedure lasted a mean time of 97.5 minutes (65-135 minutes). The mean fluoroscopic time was 44.5
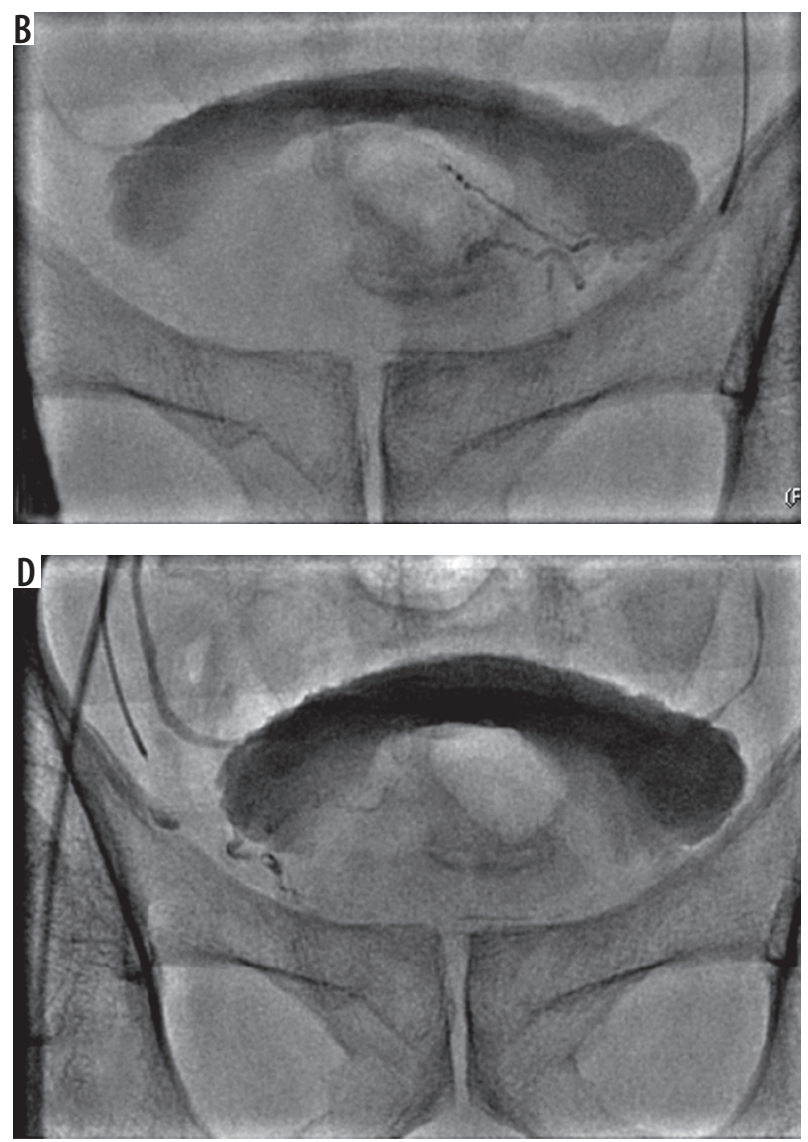

Figure 2. Selective angiography of the bilateral prostatic artery before and after embolisation. A, C) Identification of the arterial vessels of the both halves of the gland. B, D) Postembolisation follow-up angiography showing interruption of flow in both the left prostatic arteries

minutes (27-62 minutes) and iodinated contrast usage was $100 \mathrm{ml}$ (75-125 ml). No more than one vial of 100-200-microns polyvinyl alcohol particles (PVA) was used in all our cases.

None of our patients experienced any significant postprocedure complications besides mild discomfort on immediate post-procedure follow-up, which subsided with analgesics.

All our patients were followed up at one month, three months, six months, and 12 months, and the prostate volume, post void residue, IPSS, and QOL scores were recorded at each encounter. At the 12-month follow-up we found that there was a mean prostate volume reduction of $22 \%$ and mean IPSS reduction of $30 \%$ (Table 1 ). Clinical outcomes of the treatment are summarised in Table 1. We noted a downward trend in prostate volume, post void residue, IPSS, and QOL scores to a satisfactory level (Figure 4).

\section{Discussion}

Embolisation has been an essential tool in interventional radiology for a very long time. This technique has been 

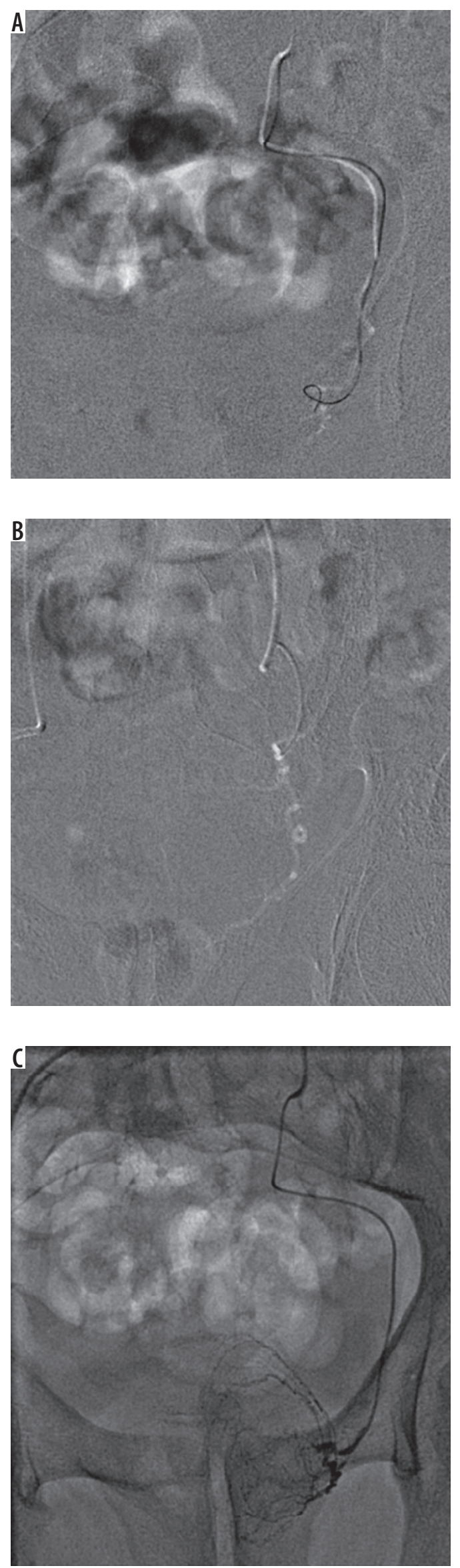

Figure 3. Use of Road Map Angiography in selective catheterisation of left prostatic arteries now utilized to treat the prostate gland. Prostate artery embolisation is a very innovative, practical, and safe alternative to the presently available surgical methods, including transurethral resection (TURP) and open prostatectomy. TURP is a gold standard procedure; however, it is associated with complications in about $20 \%$ of patients [5]. PAE is based on the principle of distal occlusion of arterial supply to the prostate gland, which causes an ischaemic response and a reduction in the prostate volume. This reduction in prostate volume causes a reduction in lower urinary tract symptoms, which includes frequency, incomplete bladder emptying, weak stream, intermittency, nocturia urgency, and straining. The International Prostate Symptom Scoring (IPSS) system is used to grade complaints in BPH. Each LUTS symptom is assigned a score of 0-5 points based on severity.

Detailed evaluation of the prostate artery in the pre-procedure period is essential in understanding the anatomy. The origin of a prostate artery can have variability. Pisco et al. [13] in their paper suggest the use of contrast angiography of the pelvic vasculature for assessment of anatomy and technical challenges, which we performed and found highly useful as a pre-procedure workup in our study. The Yamaki classification divides the internal lilac divisions into four groups: group A, B, C, and D [14]. Group A, the internal lilac divides them further into superior gluteal artery and gluteal-pudendal trunk.

Group B, divides again into internal pudendal artery and the common gluteal trunk. Group C is trifurcation, and group D is highly rare with a common trunk for the superior gluteal and internal pudendal arteries and the inferior gluteal artery. The prostatic artery mainly arises from the internal pudendal artery, superior vesical artery, or obturator artery $[15,16]$. Elderly patients have elongated and tortuous vessels with atheromatous plaque, which may cause occlusion or stenosis. A good review of the prostate artery anatomy leads to super-selective catheter placement with reduced chances of undesired ischaemic complications due to arterial anastomosis.

We noted a mean prostate volume reduction of $22 \%$, which was almost consistent with Pisco et al., who reported a $26.5 \%$ reduction in the prostate volume [17]. We noted a direct relationship between prostate volume reduction and clinical symptoms, which was, however, not demonstrated in their study. This observation is mostly because of the smaller patient pool and warrants a larger sample size.

Prostate artery embolisation has several advantages over surgical therapy, including its minimally invasive nature requiring a single femoral artery puncture. The procedure is well tolerated under conscious sedation and can be performed as a day-care procedure. Bladder catheterisation can be completely avoided. Complications such as transurethral resection syndrome, urethral stricture, contracture of the bladder neck, and bleeding can be completely avoided. 
Table 1. Outcome of the procedure

\begin{tabular}{|l|c|c|c|}
\hline Parameter & Pre-embolisation & Post-embolisation & Interpretation \\
\hline IPSS & 20 points \pm 1.5 & 10 points \pm 1.58 & Clinical improvement by 10 points \\
\hline QOL & 5 points \pm 0.54 & 1 point \pm 0.8 & Improvement in quality of life by 4 points \\
\hline Prostate volume & $74.25 c c \pm 28.6$ & $34.25 \mathrm{cc} \pm 8.26$ & Prostate volume reduction of $22 \%$ \\
\hline Postvoid residue & $54.5 \mathrm{ml} \pm 16.9$ & $30.25 \mathrm{ml} \pm 3.68$ & Improvement in postvoid urinary retention \\
\hline
\end{tabular}
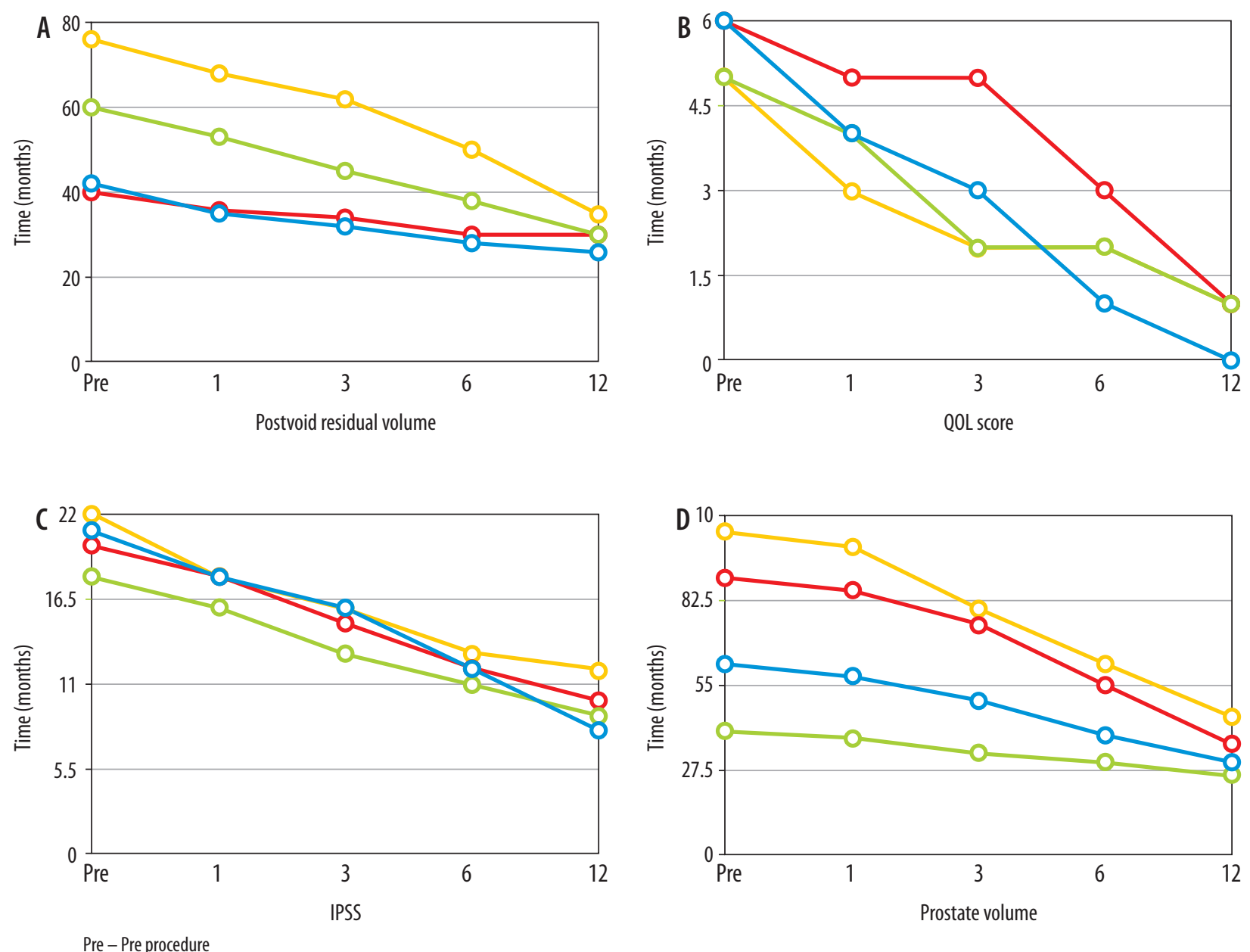

Figure 4. Changes in postvoid residual volume (A), QOL score (B), IPSS (C) and prostate volume (D) in all four patients as represented by four different colors

Post procedure the patient can be discharged after brief observation (about 5-6 hours), with analgesics for 2-3 days. Complications are rare with PAE. All our cases were discharged by the next day and did not complain of significant complications.

Our study has several shortcomings, which include: a small number of patients, short-term follow-up, and the lack of comparison with surgical techniques such as TURP. Further studies should be performed with a larger patient population to assess its true potential.

\section{Conclusions}

In this early experience, we found that prostate artery embolisation is a highly practical, feasible, and safe procedure with fascinating intermediate outcomes. Although our findings are following the existing literature, the numbers remain small because of the outreach.

\section{Conflict of interest}

The authors report no conflicts of interest. 


\section{References}

1. Wei JT, Calhoun E, Jacobsen SJ. Urologic diseases in America project: benign prostatic hyperplasia. J Urol 2005; 173: 1256-1261.

2. Rosen RC, Giuliano F, Carson CC. Sexual dysfunction and lower urinary tract symptoms (LUTS) associated with benign prostatic hyperplasia (BPH). Eur Urol 2005; 47: 824-837.

3. Garraway WM, Collins GN, Lee RJ. High prevalence of benign prostatic hypertrophy in the community. Lancet 1991; 338: 469-471.

4. McConnell JD, Bruskewitz R, Walsh P, et al. The effect of finasteride on the risk of acute urinary retention and the need for surgical treatment among men with benign prostatic hyperplasia. Finasteride Long-Term Efficacy and Safety Study Group. N Engl J Med 1998; 338: 557-563.

5. Thomas AW, Cannon A, Bartlett E, et al. The natural history of lower urinary tract dysfunction in men: minimum 10- year urodynamic follow up of transurethral resection of prostate for bladder outlet obstruction. J Urol 2005; 174: 1887e91.

6. Varkarakis J, Bartsch G, Horninger W. Long-term morbidity and mortality of transurethral prostatectomy: a 10-year follow-up. Prostate 2004; 58: 248-251.

7. Dasgupta R, Patel A. Electrosurgery of the Prostate: Improvements in Electrosurgical Unit, Transurethral Vaporization of the Prostate and Bipolar Resection. Smith's Textbook of Endourology. 3rd ed. Wiley 2012.

8. Lourenco T, Pickard R, Vale L, et al. Minimally invasive treatments for benign prostatic enlargement: systematic review of randomized controlled trials. BMJ 2008; 337: 1662.

9. Mauro MA. Can hyperplastic prostate follow uterine fibroids and be managed with transcatheter arterial embolization? Radiology 2008; 246: 657-658.
10. DeMeritt JS, Elmasri FF, Esposito MP, Rosenberg GS. Relief of benign prostatic hyperplasia-related bladder outlet obstruction after transarterial polyvinyl alcohol prostate embolization. J Vasc Interv Radiol 2000; 11: 767-770.

11. Carnevale FC, Antunes AA, da Motta Leal Filho JM, et al. Prostatic artery embolization as a primary treatment for benign prostatic hyperplasia: preliminary results in two patients. Cardiovasc Intervent Radiol 2010; 33: 355e61.

12. Ray AF, Powell J, Speakman MJ, et al. The UK ROPE Study: efficacy and safety of prostate artery embolisation for benign prostatic hyperplasia. An observational study and propensity matched comparison with transurethral resection of the prostate. BJU Int 2018; 122: $270-282$

13. Pisco JM, Rio Tinto H, Campos Pinheiro L, et al. Embolisation of prostatic arteries as treatment of moderate to severe lower urinary symptoms (LUTS) secondary to benign hyperplasia: results of shortand mid-term follow-up. Eur Radiol 2013; 23: 2561-2572.

14. Yamaki K, Saga T, Doi Y, et al. A statistical study of the branching of the human internal iliac artery. Kurume Med J 1998; 45: 333-340.

15. Bilhim T, Pisco JM, Furtado A, et al. Prostatic arterial supply: demonstration by multirow detector angio $\mathrm{CT}$ and catheter angiography. Eur Radiol 2011; 21: 1119-1126.

16. Bilhim T, Pisco JM, Rio Tinto H, et al. Prostatic arterial supply: anatomical and imaging findings relevant for selective arterial embolization. J Vasc Interv Radiol 2012; 23: 1403-1415.

17. Pisco JM, Campos Pinheiro L, Bilhim T, et al. Prostatic arterial embolization to treat benign prostatic hyperplasia. J Vasc Interv Radiol 2011; 22: 11-19. 